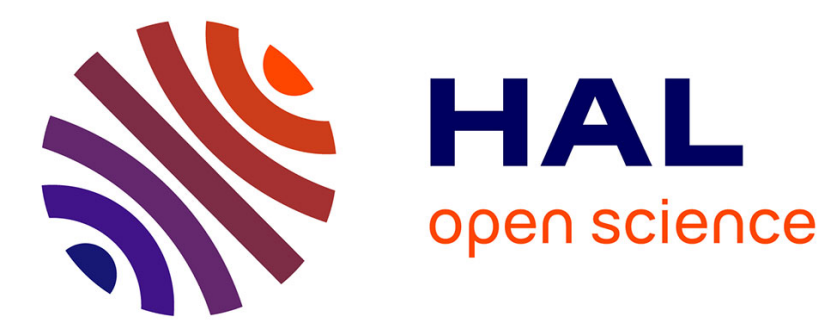

\title{
Backscattering in monomode periodic waveguides
}

\author{
Bing Wang, Simon Mazoyer, Jean-Paul Hugonin, Philippe Lalanne
}

\section{To cite this version:}

Bing Wang, Simon Mazoyer, Jean-Paul Hugonin, Philippe Lalanne. Backscattering in monomode periodic waveguides. Physical Review B: Condensed Matter and Materials Physics (1998-2015), 2008, 78 (24), pp.245108. 10.1103/PhysRevB.78.245108 . hal-00566657

\section{HAL Id: hal-00566657 \\ https://hal-iogs.archives-ouvertes.fr/hal-00566657}

Submitted on 4 Apr 2016

HAL is a multi-disciplinary open access archive for the deposit and dissemination of scientific research documents, whether they are published or not. The documents may come from teaching and research institutions in France or abroad, or from public or private research centers.
L'archive ouverte pluridisciplinaire HAL, est destinée au dépôt et à la diffusion de documents scientifiques de niveau recherche, publiés ou non, émanant des établissements d'enseignement et de recherche français ou étrangers, des laboratoires publics ou privés. 


\title{
Backscattering in monomode periodic waveguides
}

\author{
B. Wang, S. Mazoyer, J. P. Hugonin, and P. Lalanne* \\ Laboratoire Charles Fabry de l'Institut d'Optique, CNRS, Univ Paris-Sud, Campus Polytechnique, RD 128, 91127 Palaiseau Cedex, \\ France
}

(Received 15 April 2008; revised manuscript received 27 September 2008; published 8 December 2008)

\begin{abstract}
The transport properties of photonic periodic (monomode) waveguides in the presence of realistic fabrication errors are analyzed. They are governed by out-of-plane loss and backscattering. We derive a closed-form expression for the mean-free path that characterizes the transition between the ballistic and localization transport regimes in these waveguides. In agreement with earlier works, the mean-free path is found to be dominantly affected by backscattering for small group velocities. The predictions are quantitatively supported by fully vectorial computational results obtained for two-dimensional periodic waveguides. Three-dimensional (3D) structures, such as single-row-defect photonic-crystal waveguides, have also been analyzed and are shown to provide moderate backscattering in comparison to other 3D waveguides. But in all test cases, we find that the mean-free path is critically small, even for moderately small group velocities of $c / 50$ and for up-to-date fabrication nanofacilities.
\end{abstract}

DOI: 10.1103/PhysRevB.78.245108

PACS number(s): 42.70.Qs, 42.25.Fx, 42.79.Gn, 81.16.Nd

\section{INTRODUCTION}

Planar two-dimensional (2D) photonic-crystal (PhC) slabs have attracted much attention recently as a possible platform for densely integrated photonic circuits and different photonic functionalities. Engineering the photonic dispersion relationship might provide unique opportunities for integrated photonics especially in the slow light regime where new compact devices, such as delay lines, optical switches, or Raman lasers, are currently the subject of intense research. ${ }^{1-5}$ There are many important issues in relation with the use of slow light for optical processing, such as light injection ${ }^{6,7}$ or group-velocity-dispersion-management problems. ${ }^{3,8}$ A critical issue is the extrinsic loss that results from inevitable fabrication errors and that prevent full periodicity to be realized in practice. This implies that even for periodic waveguides operating with a truly guided Bloch mode below the light line of the cladding, light transport is accompanied by outof-plane and backscattering losses, like in $z$-invariant waveguides. ${ }^{9-11}$ There is no motivation for exploring the slow light regime if any advantage is immediately counteracted by excessive losses. In this context, several authors ${ }^{12-14}$ have suggested that the extrinsic losses in periodic waveguides are drastic near the band edge, where the group velocity vanishes, and may even be responsible for light localization. $^{15}$

In this work, we numerically study the transport of light in monomode periodic waveguides, focusing on the backscattering loss issue. Through a perturbative first-Bornapproximation theory similar to that used in Refs. 12, 14, 16, and 17 , we derive a universal coefficient $\rho$ that quantifies the mean-free path induced by backscattering in periodic waveguides (see Sec. II). This coefficient that allows comparisons of the performance of various waveguides scales as the square of the group index and can be calculated with virtually any Bloch-mode solver. It solely depends on the statistical disorder correlation function and on the slow Bloch-mode field distribution on the scatterers (Born approximation). In Sec. III, we validate the analytical formula for $\rho$ by comparing the formula predictions with computational results obtained for $2 \mathrm{D}$ periodic waveguides with a fully vectorial Fourier modal method. ${ }^{18,19}$ The comparison allows us to check the accuracy of the $\rho$ formula and to discuss the transition from the ballistic to the localized transport regimes, where multiple scattering becomes predominant. In Sec. IV, we calculate the $\rho$ coefficient of various 2D and three-dimensional (3D) geometries, such as single-rowdefect waveguides in PhC slabs. We find that backscattering is a very critical issue even for small disorders and for operation at moderate group velocities. Section V summarizes the work and offers open remarks.

\section{BACKSCATTERING ANALYTICAL MODEL}

Let us start by considering Fig. 1 related to light scattering in a periodic waveguide with imperfections. The unperturbed waveguide defined by its permittivity and permeability $\varepsilon$ and $\mu=\mu_{0}$ is assumed to sustain a forward-propagating truly guided Bloch mode denoted by $\Phi^{(1)}=\left|\mathbf{E}^{(1)}, \mathbf{H}^{(1)}\right\rangle$, being a pseudoperiodic function of the $z$ coordinate, $\boldsymbol{\Phi}^{(1)}(\mathbf{r}+a \mathbf{z})$ $=\exp \left(j k_{1} z\right) \boldsymbol{\Phi}^{(1)}(\mathbf{r})$ with $\operatorname{Im}\left(k_{1}\right)=0 ; a$ being the period along the $z$ direction. As this fundamental mode propagates through the perturbed section, it is backscattered into the backward propagating Bloch mode $\boldsymbol{\Phi}^{(-1)}=\left|\mathbf{E}^{(-1)}, \mathbf{H}^{(-1)}\right\rangle$ (backscattering loss $R$ ) and it is radiated in the cladding (out-of-plane losses $P)$, like in classical $z$-invariant waveguides. ${ }^{9}$ The scattering problem defines two quantities $r\left(|r|^{2}=R\right)$ and $t\left(|t|^{2}=T\right)$ that correspond to the modal reflection and modal transmission

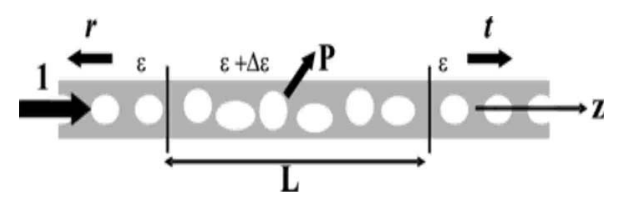

FIG. 1. Extrinsic loss in a periodic waveguide. The imperfect region $(\Delta \varepsilon)$ of length $L$ induces backscattering $R=|r|^{2}$ and out-ofplane losses $P$. This results in a lowered transmission $T=|t|^{2}$. 
coefficients, respectively. We denote by $\Delta \varepsilon$ the dielectric perturbation in the imperfect section of length $L$ and by $\boldsymbol{\Phi}$ $=|\mathbf{E}, \mathbf{H}\rangle$ : the solution of Maxwell's equations. In the orthogonal Cartesian coordinate $(x, y, z)$ system, $\boldsymbol{\Phi}$ satisfies the curl Maxwell's equations at a given frequency $\omega(\omega / c=2 \pi / \lambda)$,

$$
\boldsymbol{\nabla} \times \mathbf{E}=j \omega \mu(\mathbf{r}) \mathbf{H}, \quad \boldsymbol{\nabla} \times \mathbf{H}=-j \omega \varepsilon(\mathbf{r}) \mathbf{E}-j \omega \Delta \varepsilon(\mathbf{r}) \mathbf{E},
$$

where $j^{2}=-1$. Equation (1) shows that the imperfections act as current sources proportional to $\Delta \varepsilon$ and to the perturbed field. The infinitesimal source $-j \omega \Delta \varepsilon(\mathbf{r}) \mathbf{E}(\mathbf{r}) \mathrm{d} \mathbf{r}$ located at $\mathbf{r}$ $=(x, y, z)$ contributes to the modal Bloch-mode reflection coefficients by an infinitesimal way. Following the formalism developed in Ref. 20 that relies on the Lorentz reciprocity theorem for periodic waveguides, we have

$$
r=-(\omega / 4) \int d \mathbf{r} \Delta \varepsilon(\mathbf{r}) \mathbf{E}^{(1)}(\mathbf{r}) \cdot \mathbf{E}(\mathbf{r}),
$$

where the integral runs over the perturbed region. Note that for Eq. (2) to be valid, the Bloch mode $\boldsymbol{\Phi}^{(1)}$ has to be normalized so that its power flow through any transverse waveguide cross section is unitary. ${ }^{20}$ Equation (2) is exact but it supposes that the scattered field be known in the perturbed region. A fully vectorial calculation of $\mathbf{E}(\mathbf{r})$ is a challenging task that would require computations in three dimensions with a high resolution in order to capture the small disorder imperfections. However, such computations can be performed in two dimensions with a high accuracy and will be used in Secs. III and IV to check the accuracy of the model and its domain of validity.

To keep the treatment at a simple level, we have used a modified first-order Born approximation to derive a semianalytical expression for the backscattered intensity in the weak perturbation regime (ballistic transport). The analysis that shares many features with earlier works ${ }^{12,14,16,17}$ is provided in the Appendix for the sake of clarity. In the limit of small disorders and small group velocities, we obtain [see Eq. (A6) in the Appendix]

$$
\begin{aligned}
\langle R\rangle= & (\pi / 2)^{2}\left(\varepsilon_{b}-\varepsilon_{h}\right)^{2}(\sigma / \lambda)^{2}(L / a) n_{g}^{2} \iint_{\text {cell }} \Gamma\left(\mathbf{r}_{1}, \mathbf{r}_{2}\right) \\
& \times \alpha^{\prime}\left(\mathbf{r}_{1}\right) \alpha^{\prime *}\left(\mathbf{r}_{2}\right) \mathrm{d} \mathbf{r}_{1} \mathrm{~d} \mathbf{r}_{2},
\end{aligned}
$$

where the integral runs over the etched surfaces of the unit cell. All quantities are defined in the Appendix. $n_{g}$ is the Bloch-mode group index, $\sigma$ is the disorder standard deviation, $\Gamma\left(\mathbf{r}_{1}, \mathbf{r}_{2}\right)$ is the normalized displacement statistical correlation function, $\sigma^{2} \Gamma\left(\mathbf{r}_{1}, \mathbf{r}_{2}\right)=\left\langle\Delta l\left(\mathbf{r}_{1}\right) \Delta l\left(\mathbf{r}_{2}\right)\right\rangle$ with $\Delta l(\mathbf{r})$ the local deformation, $\varepsilon_{b}$ and $\varepsilon_{h}$ are the relative permittivities of the etched material and of the etched inclusion, and $\alpha^{\prime}(\mathbf{r})$ $=\boldsymbol{E}_{T}^{(1)}(\mathbf{r}) \cdot \boldsymbol{E}_{T}^{(1)}(\mathbf{r})+\left(\varepsilon_{b} \varepsilon_{h}\right)^{-1} \boldsymbol{D}_{N}^{(1)}(\mathbf{r}) \cdot \boldsymbol{D}_{N}^{(1)}(\mathbf{r}), \boldsymbol{E}_{T}^{(1)}$ and $\boldsymbol{D}_{N}^{(1)}$ being the normalized tangential electric and normal displacement vectors of the Bloch mode $\boldsymbol{\Phi}^{(1)}$ computed at the operating point (mainly the Brillouin-zone edge in this work). Equation (3) relies on a number of classical approximations (weak disorder on interfaces only, i.e., no volume disorder, statistical disorder correlation length smaller than the wave-
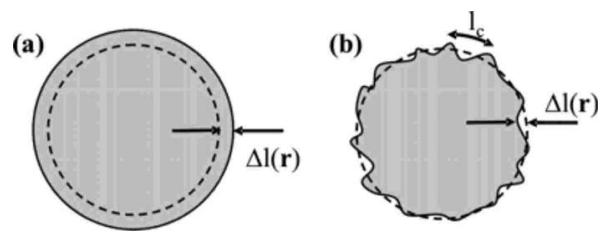

FIG. 2. Statistical disorder hole model. The local deformation is denoted $\Delta l(\mathbf{r})$. (a) Simplified size disorder model $\Delta l(\mathbf{r})=\Delta l$ used in this work. (b) More realistic model with the roughness correlation length $l_{c}$. The relationship between the loss predictions obtained with the two models is discussed at the end of Sec. II.

guide periodicity) and allows us to define a dimensionless coefficient $\rho$

$$
\rho=\lim _{\sigma \rightarrow 0} \frac{\langle R\rangle}{(\sigma / \lambda)^{2}(L / \lambda) n_{g}^{2}},
$$

with $\rho$ given by

$$
\begin{aligned}
\rho= & (\pi / 2)^{2}\left(\varepsilon_{b}-\varepsilon_{h}\right)^{2}(\lambda / a) \iint_{\text {cell }} \Gamma\left(\mathbf{r}_{1}, \mathbf{r}_{2}\right) \\
& \times \alpha^{\prime}\left(\mathbf{r}_{1}\right) \alpha^{\prime *}\left(\mathbf{r}_{2}\right) \mathrm{d} \mathbf{r}_{1} \mathrm{~d} \mathbf{r}_{2} .
\end{aligned}
$$

Hereafter, we will be concerned by periodic waveguides composed of slits or holes. As a statistical disorder, we consider a size disorder model, ${ }^{14,16,17}$ in which the hole radii (or the slit width) are randomly varied around their mean value with a statistical distribution [see Fig. 2(a)]. We further assume that distinct etched features (holes or slits) have fully independent deformations $\left[\Gamma\left(\mathbf{r}_{1}, \mathbf{r}_{2}\right)=0\right.$ if $\mathbf{r}_{1}$ and $\mathbf{r}_{2}$ are relative to two different holes] even within a single unit cell and that the correlation is perfect in the vertical direction. Depending on the fabrication tool and on the specific waveguide geometry, the two last hypotheses may be valid or not. However, we believe that they are reasonable for many geometries (such as single-row-defect $\mathrm{PhC}$ waveguides or ridges with slits) that are fabricated by electron-beam writing and etching techniques. Thus, the normal local deformation $\Delta l(\mathbf{r})$ becomes constant for every etched feature, $\Delta l(\mathbf{r})=\Delta l$ with $\langle\Delta l\rangle=0$ and $\left\langle\Delta l^{2}\right\rangle=\sigma^{2}$, and the expression of $\rho$ in Eq. (5) becomes

$$
\rho=(\pi / 2)^{2}\left(\varepsilon_{b}-\varepsilon_{h}\right)^{2}(\lambda / a) \sum_{i}\left|\int_{S_{i}} \alpha^{\prime}(\mathbf{r}) \mathrm{d} \mathbf{r}\right|^{2},
$$

which is an incoherent sum over the holes or slits surfaces $S_{i}$ contained in the unit cell. It is important to have in mind that Eq. (6) does not take into account multiple scattering. For small disorders, this approximation is likely to be valid within a single unit cell; but as photons propagate into the waveguide over long distances, multiple scattering inevitably takes place as we should see in Sec. III.

Equation (6) holds for randomly varying hole radii. This disorder choice is motivated for its simplicity, especially for comparing our model with fully vectorial calculation results that can only be obtained for simple local deformations. Actually, a more realistic disorder model is shown in Fig. 2(b), where we introduce the roughness correlation length $l_{c}$. It is 
interesting to briefly identify what is the model predictions for these more realistic deformations; this is straightforward if one assumes that the Bloch-mode field is nearly constant all around the hole circumference. Then the incoherent summation in Eq. (6) takes place over the correlation length and not over the entire circumference $2 \pi r_{h}$ as it is the case for the size disorder model. Consequently, the actual $\rho$ factor for the realistic model is found to be $2 \pi r_{h} / l_{c}$ times smaller than that obtained with the simplified model, provided that the same disorder standard deviation $\sigma$ is used for the two models. While it is very difficult in practice to estimate accurately the roughness disorder even with direct measurements, it is interesting to put realistic numbers. For a $W 1 \mathrm{PhC}$ waveguide with hole radius $r_{h} \approx 120 \mathrm{~nm}$ (see Sec. IV) and for a correlation length $l_{c}=70 \mathrm{~nm}, 2 \pi r_{h} / l_{c} \approx 9$, implying that our simplified model overestimates $\rho$ by almost an order of magnitude. In different words, we could also conclude that the amount of backscattering that is predicted with the simplified roughness model for a disorder standard deviation $\sigma$ $=1 \mathrm{~nm}$ actually corresponds to that predicted with the more realistic model for $\sigma=3 \mathrm{~nm}$. For experiments performed with periodic waveguides with lengths of several millimeters, it is conceivable that neither the simplified disorder model nor the realistic one strictly applies over the entire waveguide. Then one may consider the model as a tool to infer trends that allow comparing different geometries or technologies rather than as an accurate description providing absolute values.

\section{FROM THE BALLISTIC TO THE LOCALIZED TRANSPORT REGIMES}

In order to verify the validity and the accuracy of Eqs. (4) and (6), we have performed 2D calculations for periodic waveguides (periodicity $a=350 \mathrm{~nm}$ ) composed of slits etched in a semiconductor $(n=3.5)$ planar slab suspended in air [see Fig. 3(a)]. As a disorder model, we consider that the slit widths are randomly varied around a mean value $(\langle w\rangle$ $=150 \mathrm{~nm})$ according to a Gaussian distribution with standard deviation $\sigma$ and that they are all independent. Using the aperiodic-Fourier modal method (a-FMM), ${ }^{18,19}$ we have calculated the Bloch-mode transmittance $T=|t|^{2}$ and reflectance $R=|r|^{2}$ of finite-length waveguide sections in the vicinity of the first valence band, where $v_{g}$ vanishes and where the fundamental Bloch mode is lossless. The a-FMM, a fully vectorial method, is a generalization of Fourier modal methods ${ }^{21}$ used in grating theories to handle nonperiodic situations by an artificial periodization via the introduction of perfectly matched layers ${ }^{18}$ or of complex nonlinear coordinate transformations. ${ }^{19}$ To compute the modal transmission or reflection coefficients of the fundamental Bloch mode, we use the Bloch-mode orthogonality and reciprocity relationships. ${ }^{20}$ For the two-dimensional geometries considered in that section, a large number of Fourier harmonics (we use 201 harmonics) are retained for the computation and high accuracy is achieved. The numerical error bar is within the thickness of the lines used to show the calculated data. The calculations have been performed for the first conduction and valence bands and for both TE ( $E$ field parallel to
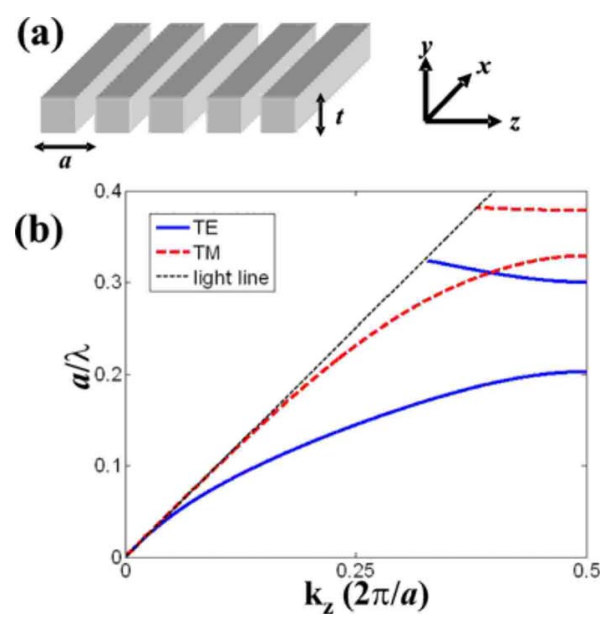

FIG. 3. (Color online) 2D waveguide geometry considered in this work. (a) Sketched of the geometry composed of air gooves (width is $150 \mathrm{~nm})$ in a semiconductor $(n=3.5)$ slab membrane $(t$ $=200 \mathrm{~nm}$ ) in air. The period is $a=350 \mathrm{~nm}$. Note that the geometry is invariant in the $x$ direction. (b) Band diagram of the fundamental guided mode for TE (solid blue) and TM (dashed red) polarizations.

the slit) and TM ( $H$ field parallel to the slit) polarizations [see Fig. 3(b) for details].

The solid lines in Fig. 4 represent the mean values of the backscattering $\langle R\rangle$ averaged over an ensemble of 1000 independent random disorder realizations, as a function of the normalized length $L$ for various $n_{g}$ and $\sigma$ values. Figures 4(a) and 4(b) are obtained for TE polarization and Figs. 4(c) and 4(d) hold for TM polarization. All plots correspond to calculation performed for the first valence band. In Figs. 4(a) and $4(\mathrm{c}), \sigma=1 \mathrm{~nm}$ and four values of the group index $n_{g}=25,50$,

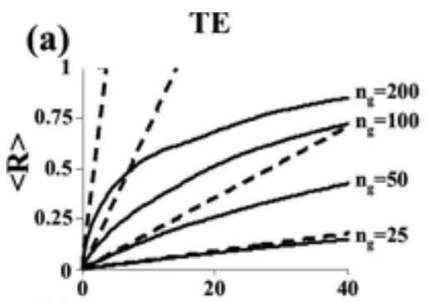

(b)
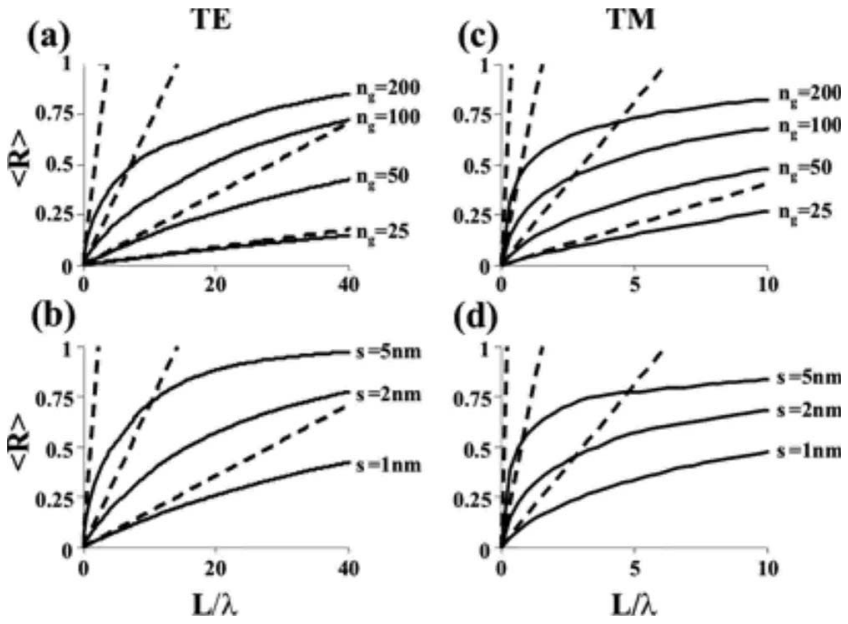

(d)

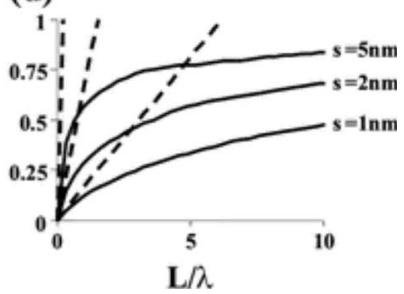

FIG. 4. a-FMM calculations (solid curves) and model predictions (dashes lines) both show a drastic effect of disorder on backscattering. The results are obtained for the periodic waveguide shown in Fig. 3 and for the first valence band. (a) $\sigma=1 \mathrm{~nm}$ for four values of the group index $n_{g}=25,50,100$, and 200. (b) $n_{g}=50$ and $\sigma=1,2$, and $5 \mathrm{~nm}$. (a) and (b) hold for TE polarization. (c) and (d): same as (a) and (b) for TM polarization. The a-FMM fully vectorial results for $\langle R\rangle$ are obtained for slit-width disorders obeying a normal distribution and are averaged over 1000 independent statistical realizations of disorders. 
(a)

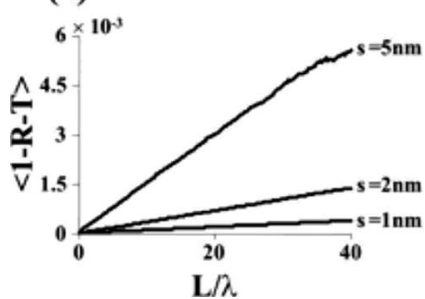

(b)

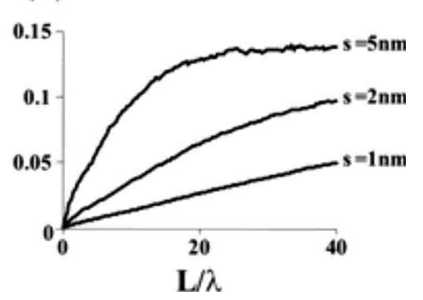

FIG. 5. Same as in Figs. 4(b) and 4(d) but for the out-of-plane losses $\langle 1-R-T\rangle$. Note the different scales in (a) and (b). (a): TE. (b): TM.

100, and 200 are considered. Figures 4(b) and 4(d) are obtained for $n_{g}=50$ and for three values of $\sigma, \sigma=1,2$, and 5 $\mathrm{nm}$. For all curves, the average reflectance first scales linearly with the normalized length before saturating, showing the transition between the ballistic and the diffuse regimes.

The dashed lines represent the linear relationships $\langle R\rangle$ $=\rho n_{g}^{2}(\sigma / \lambda)^{2} L / \lambda$, with the $\rho$ values computed from Eq. (6), $\rho=21$ for TE and $\rho=73$ for TM. It is noticeable that the dashed lines represent the slope at the origin $L=0$ of all the a-FMM data. In our opinion, this represents a strong evidence of the validity of Eq. (6), at least for 2D periodic waveguides, showing that the backscattering loss scales as $n_{g}^{2}$ (Ref. 12) and as $(\sigma / \lambda)^{2}$ in the ballistic regime $(R \ll 1$ and $T \approx 1$ ), where the Born approximation is valid.

In Fig. 5, we show the averaged out-of-plane scattering losses $\langle 1-R-T\rangle$ as a function the normalized length for $n_{g}$ $=50$ and for three values of $\sigma$, as in Figs. 4(b) and 4(d). Again the plots are obtained for the valence bands. The TE polarization case [Fig. 5(a)] corresponds to a situation for which the out-of-plane losses are much smaller than the backscattering loss. In this regime, $\langle 1-R-T\rangle$ scales linearly with $L / \lambda$ and with $n_{g}$, letting us suspect that one may define a coefficient $\gamma$ (similar to $\rho$ ) for the out-of-plane losses, $\langle 1-R-T\rangle=\gamma n_{g}(\sigma / \lambda)^{2} L / \lambda$. Within the Born approximation, the calculation of $\gamma$ requires that the coupling of the incident guided state $\boldsymbol{\Phi}^{(1)}$ with the continuum of radiation states be accurately taken into consideration. Although this calculation can be performed, ${ }^{22,12}$ it is much more intricate than that of $\rho$ and analytical expressions cannot be obtained. For the TM polarization case [Fig. 5(b)], the out-of-plane losses are significantly larger than those obtained for TE polarization. The photon transport shows an intricate behavior that combines backscattering and out-of-plane losses. For instance, for $\sigma$ $=5 \mathrm{~nm}$, Figs. 4(d) and 5(b) evidence that a saturation regime is obtained for short propagation distances, where $\approx 13 \%$ of the incident photons are lost in radiation states while the other $87 \%$ are backscattered. As shown by other computational data (not shown here for the sake of compactness), the relative fractions of photons lost by out-of-plane radiation or by backscattering in the saturation regime depends on $n_{g}$ and $\sigma$ in an intricate way; but the general trend is that as $n_{g}$ increases, backscattering dominates and $\langle R\rangle$ tends to $1 .^{22}$

In all plots, we observe that the averaged backreflection $\langle R\rangle$ and the out-of-plane losses $\langle 1-R-T\rangle$ first scale linearly with $L$ in agreement with Eq. (6). For large $L$ values, multiple scattering occurs and both quantities saturate. This char- acterizes the transition from ballistic to localization transports, which takes place at typical characteristic lengths equal to the effective mean-free path $\ell$. The latter is impacted both by backreflection and by out-of-plane loss and can be defined as the length for which $\langle T\rangle \approx 1 / 2$. Neglecting the contribution of out-of-plane scattering $\gamma n_{g}(\sigma / \lambda)^{2}$, the mean-free path becomes the length for which $\langle R\rangle \approx 1 / 2$, and is approximately given by

$$
\ell=0.5 \lambda /\left[\rho n_{g}^{2}(\sigma / \lambda)^{2}\right] .
$$

Note that this expression neglects the out-of-plane losses and multiple scattering. Thus it only provides an approximate estimation for $\ell$. Actually, this upper bound is found to be fairly small. For instance, for $\sigma=1 \mathrm{~nm}$ and for a moderate group index of 50, the effective mean-free paths are as small as $30 \lambda$ and $3 \lambda$ for TE and TM polarizations, respectively. The ten-fold variation arises from the electric-field distribution in the slit interfaces. For the first valence band and for TE polarization, the electric field is strongly localized inside the ridges and is thus rather weak on the vertical slit interfaces. The situation is the opposite for the other polarization because the electric field is dominantly confined in the air slits.

\section{THREE-DIMENSIONAL GEOMETRIES}

While the numerical results presented so far are obtained for $2 \mathrm{D}$ geometries, the model developed in the Appendix can be used to study the effects of disorder-induced backscattering in a variety of waveguide geometries that are essentially one dimensional (1D) in nature. In our opinion, the coefficient $\rho$ that solely depends on the normalized statistical correlation function and on the fundamental Bloch mode is an important figure of merit that quantifies the effective meanfree path in periodic waveguides, at least when the radiation losses are smaller than the backscattering losses. Additionally, $\rho$ varies as $\lambda / a$, implying that if one scales all the waveguide parameters with the wavelength, $\rho$ is kept unchanged. Thus the parameter $\rho$ allows for a comparison between various periodic waveguides. A question arises whether some specific geometries offer lower backscattering losses than others. Especially, one may wonder if the single-row-defect $\mathrm{PhC} W 1$ waveguide, which is currently the subject of intense research in the slow light regime, ${ }^{23-26}$ offers low backscattering losses with respect to other periodic waveguides.

To provide a first insight into this question, we have computed the $\rho$ factor of several periodic waveguides for several Bloch modes in the first bands. The results are summarized in Fig. 6. Figure 6(a) is related to a classical $W 1$ waveguide in a semiconductor $(n=3.5)$ membrane (thickness $220 \mathrm{~nm})$ in air (hole radius $r_{h}=0.3 a$ and periodicity $a=420 \mathrm{~nm}$ ). As a disorder model, we assume that only the two hole rows surrounding the single-row defect are perturbed by a random diameter fluctuation. The $W 1$ waveguide supports three bands. The upper flat one (with a null group velocity at $\lambda$ $=1.55 \mu \mathrm{m})$ corresponds to the classical $W 1$ Bloch mode that lays within the band gap of the photonic crystal and the two other bands at lower energies correspond to index-guided modes. The $\rho$ of the upper band is found to be 29: a value 

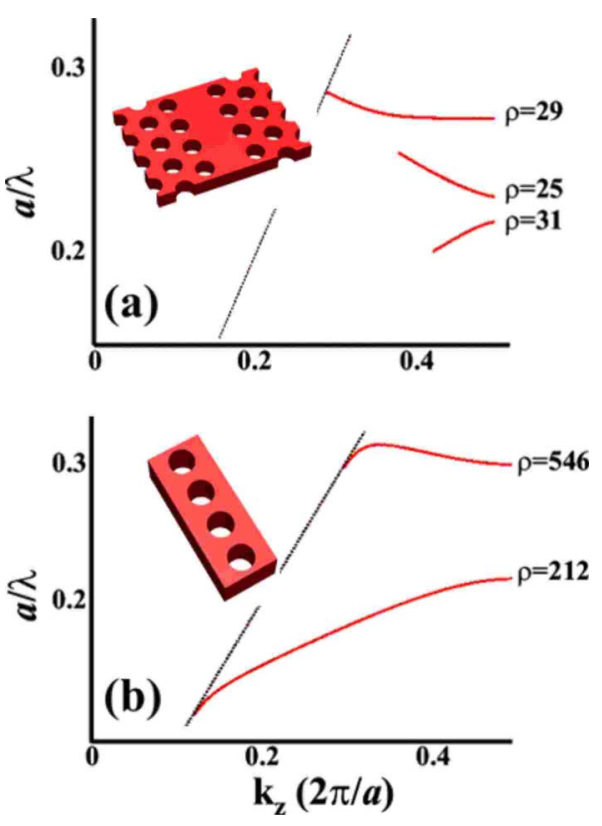

FIG. 6. (Color online) $\rho$ coefficients obtained for 3D periodic waveguide geometries and for various band edges at $k=\pi / a$. (a) $W 1$ waveguide in a semiconductor $(n=3.5)$ membrane (thickness $220 \mathrm{~nm})$ in air $\left(r_{h}=0.3 a\right.$ and $\left.a=420 \mathrm{~nm}\right)$. (b) Air-wire semiconductor waveguide (width is $500 \mathrm{~nm}$; height is $340 \mathrm{~nm}$ ) with $r_{h}$ $=0.34 a=114 \mathrm{~nm}$ and $a=335 \mathrm{~nm}$. Similar calculations have been performed for $r_{h}=50 \mathrm{~nm}$ and much smaller values for $\rho$ have been obtained $(\rho=5$ and 396 for the valence and conduction bands, respectively).

comparable to the two other ones. It is important to have in mind that the two lower band frequencies do not correspond to monomode operation since $\mathrm{PhC}$ bulk modes also exist at those frequencies. However the first-Born approximation used to derive the $\rho$ factor remains valid even for multimode waveguides, since Eq. (2) solely relies on the forward and backward propagating modes $\boldsymbol{\Phi}^{(1)}$ and $\boldsymbol{\Phi}^{(-1)}$. Note that Eq. (2) applies to all the guided modes and can be used to calculate backscattering into higher-order modes. However, multimode operation impacts backscattering into the fundamental mode as shown in Ref. 11 for $z$-invariant waveguides; but analyzing this effect requires to go beyond the first-order Born approximation and to consider multiple-scattering situations, for which photons are first scattered into higher-order modes and then backscattered into the fundamental mode. This is beyond the scope of the present work.

We have also performed calculations in two dimensions for the same geometry using an effective index of 2.83 for the refractive index of the semiconductor and a magnetic polarization parallel to the $\mathrm{PhC}$ cylinder $y$ axis. The effective index value was chosen so that the cut-off frequency of the $W 1$ waveguide at the edge of the first Brillouin zone matches that of the 3D structure. The calculated $\rho$ values are similar to those obtained with the 3D calculations ( $\rho=36$ instead of 29 for the upper band), showing that 2D calculations are quantitatively accurate although they do not take into account the electric-field component that is parallel to the cylindrical hole axis.

Figure 6(b) is related to a semiconductor $(n=3.5)$ periodic nanowire (width is $500 \mathrm{~nm}$ and height is $340 \mathrm{~nm}$ ) in air, with a hole radius $r_{h}=0.34 a$. The bands correspond to Bloch modes predominantly TE polarized (like in the $W 1$ ). The periodicity has been chosen equal to $a=335 \mathrm{~nm}$ so that $\lambda$ is equal to $1.55 \mu \mathrm{m}$ at the Brillouin-zone edge of the valence band. As for the planar waveguide with slits in TE polarization, we found that the $\rho$ value associated to the valence band is significantly smaller than that of the conduction band. The calculation of the $\rho$ values is obtained, as previously, by assuming a hole-diameter disorder model. The $\rho$ value of 212 calculated in the first valence band is significantly larger than that obtained for the $W 1$ waveguide. Similar calculations have been performed for smaller holes $(r=50 \mathrm{~nm})$, and a significant lowering of the $\rho$ factor for the valence band $(\rho=5)$ has been obtained. This reduction is also accompanied with a lowering of the band-gap width that becomes comparable to that obtained for the $W 1$ waveguide in Fig. 6(a) for the two lower branches.

It is interesting to compare the model prediction for $W 1$ waveguides with experimental results obtained at telecom wavelengths $(\lambda=1.55)$. In Ref. 13, transmission measurements performed for different lengths (from $100 \mu \mathrm{m}$ to 1 $\mathrm{mm}$ ) by the cut-back method have shown that for moderate group velocities $n_{g}=40$, the total transmission loss are as large as $\alpha=800 \mathrm{~dB} / \mathrm{cm}$. Assuming that the total attenuation is predominantly due to backscattering and that the transmission is given by $\exp \left[-\rho n_{g}^{2}(\sigma / \lambda)^{2} L / \lambda\right]$ in the localization regime, ${ }^{27}$ we obtain $\sigma=1.3 \mathrm{~nm}$ by equaling $-\rho n_{g}^{2}(\sigma / \lambda)^{2} L / \lambda$ and $\alpha$ for $\rho=29$ and $n_{g}=40$. We regard the $\sigma$ value to be physically reasonable, although it is likely to slightly underestimate the actual disorder standard deviation $\sigma=3-4 \mathrm{~nm}$, as inferred in Ref. 13 from scanning electron microscopy (SEM) pictures. But we believe that our underestimation of the disorder level basically results from the specific hole size disorder model we use (see the previous discussion at the end of Sec. II). In addition, it is important to realize that the loss measurements in Ref. 13 are performed in the multiplescattering (localized transport) regime for propagation lengths much larger than the mean-free path. For this regime, the Born approximation is no longer valid and the intricate interplay between out-of-plane and backscattering losses [see Figs. 4(d) and 5(b), for instance] are likely to render our $\rho$ analysis too simplistic. More studies are necessary to understand the transport properties in the localization regime.

\section{CONCLUSION}

Through a perturbative approach, we have derived a universal coefficient $\rho$ that quantifies the amount of backscattering in periodic waveguides. This coefficient, which solely depends on the statistical disorder model and on the slow Bloch-mode field distribution, can be easily calculated with presently available software. The model predictions have been quantitatively checked (Fig. 4) against fully vectorial computational results obtained for 2D periodic waveguides composed of slit arrays in slab waveguides. Quantitative agreements have been obtained for all bands and for short propagation distances, showing the validity of the model at least in two dimensions.

The performance of 3D single-row-defect $\mathrm{PhC}$ $W 1$-waveguides has also been analyzed (Fig. 6). Due to the 
side-located periodic holes, the backscattering losses in that waveguide are slightly smaller than those achieved for periodic waveguides with similar confinements and with fully centered holes. The gap-guiding mechanism that is a unique property of $\mathrm{PhC}$ waveguide does not appear to be crucial in the context of extrinsic losses, at least for small group velocities.

In all our simulations, we have found that backscattering due to imperfections is fairly drastic. Although the $\rho$ coefficient of $W 1$ waveguides appears modest in comparison to those of other waveguides, it is found that the mean-free path [see Eq. (7)] of these waveguides at telecommunication wavelengths is only $25 \mu \mathrm{m}$ (60 periods): a value that holds for state-of-the-art manufactured waveguides $(\sigma=1 \mathrm{~nm})$ and for $n_{g}=50$. This corresponds to a very small group delay $(\tau$ $\left.=\ell n_{g} / c\right)$ of only $5 \mathrm{ps}$. Thus one may be inclined to operate $W 1$ waveguides for propagation lengths that are larger than the effective mean-free path, as it is often the case in experimental situations. ${ }^{13,26}$ Backscattering losses (as well as outof-plane diffraction that has not been considered here) may then become prohibitive. But more importantly for signal processing, one should expect a drastic pulse broadening beyond the ballistic regime: in the localized regime, the essence of the energy transport is multiple scattering and the transmitted light experiences many backward and forward reflections as it propagates.

\section{ACKNOWLEDGMENTS}

This research is partly supported under the European contract SPLASH of the 6th EU Framework program and by the Agence Nationale de la Recherche under contract MIRAMAN of the French ANR Nano2006. We acknowledge fruitful discussions with C. Sauvan, L. C. Andreani, and J. J. Greffet.

\section{APPENDIX}

In this appendix, we develop a modified Born approximation to derive a semianalytical expression for the backscattered intensity in the weak perturbation regime. Figure 1 is related to light scattering in a periodic waveguide with imperfections. The waveguide is formed by air holes in a semiconductor background. Within a given section of length $L$, the waveguide is assumed to be perturbed by fabrication errors. Figure 7 shows an example of an exaggerated perturbation, where a circular air $\left(\varepsilon_{h}=1\right)$ hole in the semiconductor $\left(\varepsilon_{b}=3.5\right)$ background is deformed. The local deformation defined on the hole interfaces at position $\mathbf{r}$ is denoted by $\Delta l(\mathbf{r})$. The deformation defines two different regions with positive $\Delta \varepsilon(\Delta l>0)$ and negative $\Delta \varepsilon(\Delta l<0)$. With up-to-date nanofabrication facilities, roughness and disorder are in the nanometer range, while typical Bloch confinements are of the order of the wavelength. Thus, with a good approximation, the perturbed regions can be all considered as infinitely small surfaces and by assuming that the electromagnetic fields are locally constant within the deformation thickness (static limit), the volume integral over the perturbed regions in Eq. (2) can be replaced by a surface integral over all the hole

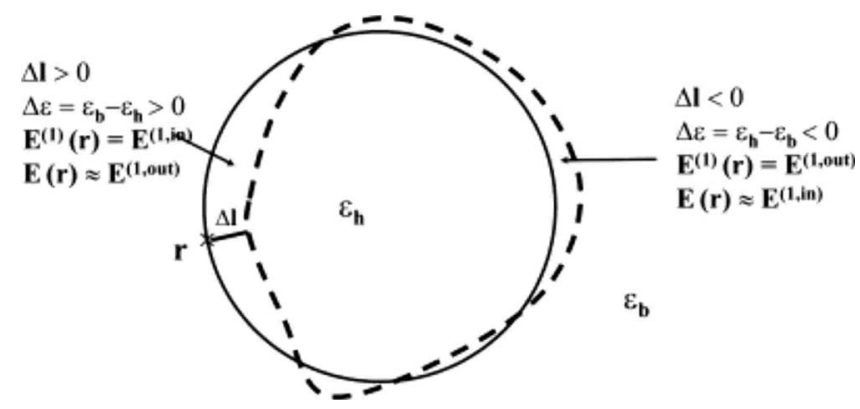

FIG. 7. Modified first-order Born approximation leading to Eq. (A2). The three main quantities $\Delta l(\mathbf{r}), \mathbf{E}^{(1)}(\mathbf{r})$, and $\mathbf{E}(\mathbf{r})$ in Eq. (A1) are all defined for the regions with positive and negative $\Delta \varepsilon$.

interfaces contained in the perturbed section of length $L$

$$
r=-(\omega / 4)\left(\varepsilon_{b}-\varepsilon_{h}\right) \int \mathrm{d} \mathbf{r} \Delta l(\mathbf{r}) \mathbf{E}^{(1)}(\mathbf{r}) \cdot \mathbf{E}(\mathbf{r}),
$$

where $\mathbf{E}^{(1)}(\mathbf{r})$ and $\mathbf{E}(\mathbf{r})$ are defined on the interface.

Referring to Eq. (A1), the first-order Born approximation merely consists in replacing the unknown perturbed electric field $\mathbf{E}(\mathbf{r})$ by the fundamental Bloch-mode (unperturbed) field $\mathbf{E}^{(1)}(\mathbf{r})$. However, this replacement has to be handled with caution. At a position $\mathbf{r}$ corresponding to a positive $\Delta l$, the unknown perturbed field $\mathbf{E}(\mathbf{r})$ is well approximated by the unperturbed Bloch-mode electric field $\mathbf{E}^{(1)}(\mathbf{r})$ calculated on the outer boundary of the hole; a quantity that we further denote by $\mathbf{E}^{(1, \text { out })}$. Similarly, for $\mathbf{r}$ in a region with $\Delta l<0$, $\mathbf{E}(\mathbf{r})$ is approximated by the fundamental Bloch-mode field $\mathbf{E}^{(1, \text { in) }}$ calculated on the inner boundary of the hole. In both cases, $\Delta l>0$ or $\Delta l<0$, the product $\mathbf{E}^{(1)}(\mathbf{r}) \cdot \mathbf{E}(\mathbf{r})$ in Eq. (A1) is equal to $\mathbf{E}^{(1, \text { in })}(\mathbf{r}) \cdot \mathbf{E}^{(1, \text { out })}(\mathbf{r})$. The relevancy of this nonstandard replacement is easily understood if one considers that the unknown field $\mathbf{E}(\mathbf{r})$ is approximately given by the fundamental Bloch mode of every perturbed cell. As we have checked with fully vectorial computational results for the backscattering $R$ of perturbed waveguides in two dimensions, this replacement that leads to a nonstandard Born approximation increases the numerical accuracy of the model predictions. $^{17}$

Thus for tangential electric-field components that are continuous across the surfaces, $\mathbf{E}^{(1, \text { out })}(\mathbf{r})=\mathbf{E}^{(1, \text { in })}(\mathbf{r})=\mathbf{E}_{T}^{(1)}(\mathbf{r})$ and $\mathbf{E}(\mathbf{r}) \cdot \mathbf{E}^{(1)}(\mathbf{r})$ is simply given by $\mathbf{E}^{(1)}(\mathbf{r}) \cdot \mathbf{E}^{(1)}(\mathbf{r})$. For normal electric-field components that are discontinuous across the interfaces, $\varepsilon_{b} \mathbf{E}^{(1, \text { out })}(\mathbf{r})=\varepsilon_{h} \mathbf{E}^{(1, \text { in })}(\mathbf{r})=\mathbf{D}_{N}^{(1)}(\mathbf{r}) ; \mathbf{D}_{N}^{(1)}$ being the displacement vector of the fundamental Bloch mode. $\mathbf{E}(\mathbf{r}) \cdot \mathbf{E}^{(1)}(\mathbf{r})$ then reduces to $\left(\varepsilon_{b} \varepsilon_{h}\right)^{-1} \mathbf{D}_{N}^{(1)}(\mathbf{r}) \bullet \mathbf{D}_{N}^{(1)}(\mathbf{r})$. To summarize, within the limit of small perturbation regions, the modified Born approximation results in

$$
r=-(\omega / 4)\left(\varepsilon_{b}-\varepsilon_{h}\right) \int \mathrm{d} \mathbf{r} \Delta l(\mathbf{r}) \alpha(\mathbf{r}),
$$

where the integral runs over the dielectric interfaces (e.g., the unperturbed hole boundaries) and the complex number $\alpha$ is given by 


$$
\alpha(\mathbf{r})=\mathbf{E}_{T}^{(1)}(\mathbf{r}) \cdot \mathbf{E}_{T}^{(1)}(\mathbf{r})+\left(\varepsilon_{b} \varepsilon_{h}\right)^{-1} \mathbf{D}_{N}^{(1)}(\mathbf{r}) \cdot \mathbf{D}_{N}^{(1)}(\mathbf{r}) .
$$

In Eq. (A3), $\mathbf{E}_{T}^{(1)}$ (respectively, $\mathbf{D}_{N}^{(1)}$ ) refers to the tangential (respectively, normal) components of the Bloch-mode electric (respectively, displacement) fields. Note that a related tensor description of roughness has been used in Refs. 14 and 17.

The mean reflectance $\langle R\rangle$ of the perturbed section of length $L$ is then easily derived from Eq. (A2), and assuming that $\Delta l$ is null on ensemble average, one gets

$$
\langle R\rangle=(\omega / 4)^{2}\left(\varepsilon_{b}-\varepsilon_{h}\right)^{2} \sigma^{2} \iint \Gamma\left(\mathbf{r}_{1}, \mathbf{r}_{2}\right) \alpha\left(\mathbf{r}_{1}\right) \alpha^{*}\left(\mathbf{r}_{2}\right) \mathrm{d} \mathbf{r}_{1} \mathrm{~d} \mathbf{r}_{2},
$$

where each integral runs over the perturbed interfaces. In Eq. (A4), $\Gamma\left(\mathbf{r}_{1}, \mathbf{r}_{2}\right)$ represents the normalized displacement correlation function defined by $\sigma^{2} \Gamma\left(\mathbf{r}_{1}, \mathbf{r}_{2}\right)=\left\langle\Delta l\left(\mathbf{r}_{1}\right) \Delta l\left(\mathbf{r}_{2}\right)\right\rangle$, with \langle\rangle a notation for ensemble averages. Hereafter, we assume that the disorder displacements induced by the fabrication errors are independent from one unit cell to the other (in the main text, we will further assume that the $\Delta l$ 's of distinct holes within a single cell are also independent). Within this reasonable hypothesis (see main text), Eq. (A4) becomes

$$
\begin{aligned}
\langle R\rangle= & (\pi c / 2)^{2}\left(\varepsilon_{b}-\varepsilon_{h}\right)^{2}(\sigma / \lambda)^{2}(L / a) \iint_{\text {cell }} \Gamma\left(\mathbf{r}_{1}, \mathbf{r}_{2}\right) \\
& \times \alpha\left(\mathbf{r}_{1}\right) \alpha^{*}\left(\mathbf{r}_{2}\right) \mathrm{d} \mathbf{r}_{1} \mathrm{~d} \mathbf{r}_{2},
\end{aligned}
$$

with $a$ being the waveguide period. The contribution of every cell adds incoherently on average and the mean backscattering scales linearly with the waveguide-section length
L. Equation (A5) that neglects multiple-scattering processes provides a semianalytical expression for the averaged backscattering. The latter solely depends on the Bloch mode of the unperturbed waveguide through $\alpha(\mathbf{r})$ and on the displacement correlation function of the statistical disorder through $\Gamma\left(\mathbf{r}_{1}, \mathbf{r}_{2}\right)$ defined within one cell.

Equation (A5) applies to any Bloch mode at any point in the dispersion diagram $\left(\omega, k_{z}\right)$. To analytically explicit the $v_{g}$ dependence of $\langle R\rangle$, we must consider a Bloch mode with a small group velocity $v_{g}$ in the vicinity of an operating point where $v_{g}$ vanishes, for instance, at the Brillouin-zone edge. Let us define by $\boldsymbol{E}^{(1)}$ and $\boldsymbol{H}^{(1)}$ the Bloch-mode electric and magnetic fields at $v_{g}=0$ normalized such that $\int_{\text {cell }}\left(\varepsilon\left|\boldsymbol{E}^{(1)}\right|^{2}\right.$ $\left.+\mu\left|\boldsymbol{H}^{(1)}\right|^{2}\right) \mathrm{d} \mathbf{r}=4 a$. Because any Bloch mode with a unitary transverse power flow satisfies $\int_{\text {cell }}\left(\varepsilon\left|\mathbf{E}^{(1)}\right|^{2}+\mu\left|\mathbf{H}^{(1)}\right|^{2}\right) \mathrm{d} \mathbf{r}$ $=4 a / v_{g}$ (Ref. 20), $\mathbf{E}_{T}^{(1)}$ or $\mathbf{D}_{N}^{(1)}$ in Eq. (A3) can approximately be written as $\mathbf{E}_{T}^{(1)}=\left(v_{g}\right)^{-1 / 2} \boldsymbol{E}_{T}^{(1)}$ or $\mathbf{D}_{N}^{(1)}=\left(v_{g}\right)^{-1 / 2} \boldsymbol{D}_{N}^{(1)}$, showing that the Bloch-mode electric and magnetic fields scale as $\left(v_{g}\right)^{-1 / 2}$. Therefore, in the limit of small disorders and small $v_{g}$ 's, we obtain

$$
\begin{aligned}
\langle R\rangle= & (\pi / 2)^{2}\left(\varepsilon_{b}-\varepsilon_{h}\right)^{2}(\sigma / \lambda)^{2}(L / a) n_{g}^{2} \iint_{\text {cell }} \Gamma\left(\mathbf{r}_{1}, \mathbf{r}_{2}\right) \\
& \times \alpha^{\prime}\left(\mathbf{r}_{1}\right) \alpha^{\prime *}\left(\mathbf{r}_{2}\right) \mathrm{d} \mathbf{r}_{1} \mathrm{~d} \mathbf{r}_{2},
\end{aligned}
$$

where $\alpha^{\prime}(\mathbf{r})=\boldsymbol{E}_{T}^{(1)}(\mathbf{r}) \cdot \boldsymbol{E}_{T}^{(1)}(\mathbf{r})+\left(\varepsilon_{b} \varepsilon_{h}\right)^{-1} \boldsymbol{D}_{N}^{(1)}(\mathbf{r}) \cdot \boldsymbol{D}_{N}^{(1)}(\mathbf{r})$ is defined for the scaled fields calculated at the operating point in the dispersion diagram, essentially the Brillouin-zone edge in this work. Note that the last approximation leading to Eq. (A6) is not strictly necessary for our analysis and that Eq. (A5) is more accurate for large $v_{g}$ values. However, Eq. (A6) that requires only a single Bloch-mode calculation at some operating point of interest allows explicitly emphasizing the group-velocity impact on the backscattered intensity.

\footnotetext{
*Corresponding author; philippe.lalanne@institutoptique.fr

${ }^{1}$ M. Soljacic and J. D. Joannopoulos, Nature Mater. 3, 211 (2004).

${ }^{2}$ T. F. Krauss, Phys. World 19 (2), 32 (2006).

${ }^{3}$ D. Mori and T. Baba, Opt. Express 13, 9398 (2005).

${ }^{4}$ Y. A. Vlasov, M. O'Boyle, H. F. Hamann, and S. J. McNab, Nature (London) 438, 65 (2005).

${ }^{5}$ M. L. Povinelli, S. Johnson, and J. Joannopoulos, Opt. Express 13, 7145 (2005).

${ }^{6}$ Y. A. Vlasov and S. J. McNab, Opt. Lett. 31, 50 (2006).

${ }^{7}$ P. Velha, J. P. Hugonin, and P. Lalanne, Opt. Express 15, 6102 (2007).

${ }^{8}$ A. Yu. Petrov and M. Eich, Appl. Phys. Lett. 85, 4866 (2004).

${ }^{9}$ D. Marcuse, Bell Syst. Tech. J. 48, 3187 (1969).

${ }^{10}$ A. Garcia-Martin, J. J. Saentz, and M. Nieto-Vesperinas, Phys. Rev. Lett. 84, 3578 (2000).

${ }^{11} \mathrm{~S}$. A. Bulgakov and M. Nieto-Vesperinas, J. Opt. Soc. Am. A 15, 503 (1998).

${ }^{12}$ S. Hughes, L. Ramunno, J. F. Young, and J. E. Sipe, Phys. Rev. Lett. 94, 033903 (2005)

${ }^{13}$ E. Kuramochi, M. Notomi, S. Hughes, A. Shinya, T. Watanabe,
}

and L. Ramunno, Phys. Rev. B 72, 161318(R) (2005).

${ }^{14}$ L. C. Andreani and D. Gerace, Phys. Status Solidi B 244, 3528 (2007).

${ }^{15}$ S. Mookherjea, J. S. Park, S.-H. Yang, and P. R. Bandaru, Nat. Photonics 2, 90 (2008).

${ }^{16}$ D. Gerace and L. C. Andreani, Opt. Lett. 29, 1897 (2004).

${ }^{17}$ S. G. Johnson, M. L. Povinelli, M. Soljacic, A. Karalis, S. Jacobs, and J. D. Joannopoulos, Appl. Phys. B: Lasers Opt. 81, 283 (2005).

${ }^{18}$ E. Silberstein, P. Lalanne, J. P. Hugonin, and Q. Cao, J. Opt. Soc. Am. A 18, 2865 (2001).

${ }^{19}$ J. P. Hugonin and P. Lalanne, J. Opt. Soc. Am. A 22, 1844 (2005).

${ }^{20}$ G. Lecamp, J. P. Hugonin, and P. Lalanne, Opt. Express 15, 11042 (2007).

${ }^{21}$ See, for instance, M. G. Moharam, E. B. Grann, D. A. Pommet, and T. K. Gaylord, J. Opt. Soc. Am. A 12, 1068 (1995).

${ }^{22}$ G. Lecamp, P. Lalanne, and J. P. Hugonin, Phys. Rev. Lett. 99, 023902 (2007).

${ }^{23}$ L. H. Frandsen, A. V. Lavrinenko, J. Fage-Pedersen, and P. I. Borel, Opt. Express 14, 9444 (2006). 
${ }^{24}$ D. Mori, S. Kubo, H. Sasaki, and T. Baba, Opt. Express 15, 5264 (2007).

${ }^{25}$ N. Ozaki, Y. Kitagawa, Y. Takata, N. Ikeda, Y. Watanabe, A. Mizutani, Y. Sugimoto, and K. Asakawa, Opt. Express 15, 7974 (2007).

${ }^{26}$ L. O'Faolain, T. P. White, D. O’Brien, X. D. Yuan, M. D. Settle, and T. F. Krauss, Opt. Express 15, 13129 (2007)

${ }^{27}$ On ensemble averages, the transmission in the localization regime decays exponentially when the system size is much larger than the localization length (Refs. 10 and 11). This condition is likely to be fulfilled in the experiments performed in Ref. 13 since the $\mathrm{PhC}$-waveguide lengths are in the millimeter range. 